Meta

Journal des traducteurs

Translators' Journal

\title{
Réflexions d'un praticien sur une étape de la formation des interprètes de conférence : approche méthodologique et pédagogique
}

\section{Benoît Kremer}

Volume 50, numéro 2, avril 2005

Processus et cheminements en traduction et interprétation Processes and Pathways in Translation and Interpretation

URI : https://id.erudit.org/iderudit/011018ar

DOI : https://doi.org/10.7202/011018ar

Aller au sommaire du numéro

Éditeur(s)

Les Presses de l'Université de Montréal

ISSN

0026-0452 (imprimé)

1492-1421 (numérique)

Découvrir la revue

Citer cet article

Kremer, B. (2005). Réflexions d'un praticien sur une étape de la formation des interprètes de conférence : approche méthodologique et pédagogique. Meta, 50(2), 785-794. https://doi.org/10.7202/011018ar

\section{Résumé de l'article}

La démarche didactique usuellement mise en oeuvre dans les premières étapes de l'enseignement de l'interprétation de conférence est décrite (mémorisation, exercices monolingues). Trois types d'exercices concrets à introduire à titre de préalable sont exposés, illustrés et soutenus par une argumentation pédagogique : la contextualisation, la hiérarchisation des idées et la création d'images mentales. 


\title{
Réflexions d'un praticien sur une étape de la formation des interprètes de conférence: approche méthodologique et pédagogique
}

\author{
BENOÎT KREMER \\ Université de Genève, Genève, Suisse \\ b.kremer@aiic.net
}

\begin{abstract}
RÉSUMÉ
La démarche didactique usuellement mise en œuvre dans les premières étapes de l'enseignement de l'interprétation de conférence est décrite (mémorisation, exercices monolingues). Trois types d'exercices concrets à introduire à titre de préalable sont exposés, illustrés et soutenus par une argumentation pédagogique: la contextualisation, la hiérarchisation des idées et la création d'images mentales.
\end{abstract}

\begin{abstract}
Teaching conference interpreting usually starts with memory and monolingual exercises. This article presents three types of exercises which ought to be introduced first and foremost, and the pedagogical rationale for these exercises is underlined. These exercises are: contextualization, prioritization of ideas and mental images.
\end{abstract}

\section{MOTS-CLÉS/KEYWORDS}

interprétation de conférence, pédagogie de l'interprétation, cognition, contexte, hiérarchisation

Qu'il soit ici permis à un praticien de l'interprétation de conférence et de son enseignement de décrire une des étapes initiales de l'apprentissage de cette discipline: la compréhension d'un message oral en vue de sa restitution. Après une brève analyse des problèmes rencontrés par les interprètes de conférence débutants et novices, nous suggérerons quelques types d'exercices permettant de faire prendre conscience aux étudiants des processus mentaux et intellectuels en jeu. Chaque exercice sera décrit en détail, puis assorti d'une évaluation de sa place et de son rôle dans la didactique de l'interprétation, ainsi que d'une description du but précis poursuivi.

Les exemples qui illustrent cette approche sont essentiellement tirés d'expériences pratiques faites ces dernières années avec des étudiants de l'École de Traduction et d'Interprétation (ÉTI) de l'Université de Genève, d'une part lors d'un cours d'initiation à l'interprétation destiné aux traducteurs en fin de formation, d'autre part lors de séances d'interprétation consécutive destinées aux étudiants en interprétation de conférence, plus précisément dans les toutes premières semaines de cours.

L'interprétation de conférence est généralement enseignée à des étudiants connaissant déjà aussi bien que possible les langues de leur combinaison linguistique. La formation, universitaire et post-gradué, vise à transmettre les compétences techniques qui assureront la maîtrise des processus d'écoute, d'analyse et de transmission du discours. Pour la décrire "à rebours», cette formation culmine généralement par l'apprentissage de l'interprétation simultanée, précédée par l'interprétation consécutive 
avec notes ${ }^{1}$; cette dernière commence généralement par des exercices sans notes, pour la préparation desquels un entraînement de la mémoire est considéré comme utile. C’est donc sur la mémorisation que s'ouvre généralement le programme de formation.

Or, il apparaît que ces exercices de mémorisation, et même les exercices de reformulation monolingue (Gile 2003) qui les suivent généralement, risquent de manquer leur but s'ils ne sont pas précédés d'autres exercices fondamentaux, que nous suggérons ici.

Le premier et principal obstacle auquel se heurte l'enseignant tient à un malentendu fondamental quant à la nature du travail attendu. "The general population often has a simplified understanding of what it means to translate. They perceive translation as a kind of lexico-semantic exercise, an act carried out on language form that requires a one-to-one substitution of target language lexical items for the lexical items of the source language » (Clifford 2001). Même si certains auteurs et enseignants affirment ne pas rencontrer généralement d'hostilité envers l'idée que l'interprète s'efforce de comprendre pour traduire (Gile 2003²) (il n'est pas impossible que Gile fasse référence à une compréhension théorique et conceptuelle, et pas nécessairement à la réalité des aptitudes manifestées en cours), la profonde trace laissée dans les esprits par un apprentissage des langues axé sur les équivalences lexicales ne doit pas être sous-estimée. Or, en traduction et a fortiori en interprétation, il ne s'agit pas de produire une correspondance littérale des mots utilisés par l'orateur, mais de restituer le sens qu'il a voulu leur donner dans le contexte précis d'une situation de communication donnée. «Bei jeder Translation wird auf eine intendierte Rezipientenschaft hin übersetzt/gedolmetscht» (Reiss 1984). Cela présuppose donc que l'interprète partage les références culturelles, sémantiques, langagières ou intellectuelles des participants (locuteur et auditeurs), sous peine, dans le meilleur des cas, de ne rien comprendre aux échanges, et dans le pire des cas, de commettre des erreurs graves.

Nous souscrivons pour notre part avec vigueur à la position prise par Ilg et Lambert: «CI [consecutive interpreting] should not be taught right from the start because it presupposes students already have the ability to carry messages across linguistic barriers. This must first be taught and acquired» (Ilg et Lambert 1996). Parmi les exercices pratiques présentés en détails comme susceptibles d'être mis en œuvre en prélude à la prise de notes figurent la mémorisation, la traduction à vue, les exercices unilingues (réexpression, restructuration, paraphrase), la préparation au multitasking, etc. ${ }^{3}$

En ce qui nous concerne, il apparaît que la maîtrise de la technique de transmission d'un message d'une langue dans une autre, décrite par Ilg et Lambert comme le préalable à tout exercice de prise de notes efficace, passe avant tout par trois étapes, souvent omises, que nous étudierons ici plus en détail: la prise de conscience de l'importance du contexte (environnement historique, géographique, culturel, nondit et sous-entendus, connaissances partagées par tous les participants mais non explicitement exposées); la sensibilisation à la nécessité de structurer les idées (hiérarchisation, liens logiques); et enfin, la faculté de se représenter mentalement, de manière aussi concrète que possible, les idées exprimées (images mentales, également appelées représentation mentale (voir Degueldre 2002). 


\section{Le contexte}

À la $X^{\text {e }}$ Conférence internationale sur le SIDA, un journaliste interrogeait un activiste de l'ANC (African National Congress) sur toutes sortes de sujets liés à la lutte contre cette pandémie. Les questions se succédaient et passaient volontiers d'un aspect du problème à un autre (considérations personnelles, politiques, médicales, etc.). Soudain, on a pu entendre le dialogue suivant:

- (le journaliste) Quelle est votre attitude envers M. Thabo Mbeki?

- (l'activiste) J'ai fini par calmer ma colère et, sans pardonner pour autant, j'accepterai sans doute un jour prochain de tourner la page.

Ce dialogue simple et direct n'emploie guère de formulations ambiguës et les phrases qui le constituent ne présentent a priori aucune difficulté de compréhension insurmontable. Mais l'interprète ne pourra maîtriser la pensée des intervenants que s'il arrive à aller au-delà des propos échangés pour reconstituer les arrière-plans qui les sous-tendent (et qui sont supposés connus de tous les participants, donc ne font l'objet d'aucun rappel explicite). Il faut savoir en effet que quelques années auparavant, le président sud-africain Thabo Mbeki avait pris une position politique controversée en affirmant que le SIDA était dû à la pauvreté et non à un virus. Une telle déclaration politique intervenait dans un pays où la diffusion de l'information la plus fondamentale sur les modes de transmission du virus était encore insuffisante et où existaient des approches de médecine traditionnelle tendant à recommander des pratiques assez proches de la superstition. Les activistes de l'époque, y compris ceux de l'ANC, et toute la communauté des chercheurs avaient vivement réagi aux propos de M. Mbeki, considérés comme une régression par rapport aux connaissances et aux objectifs de la lutte contre le SIDA. Quelque temps plus tard, face à ce tollé international et à la suite de diverses interventions, notamment de M. Nelson Mandela, M. Mbeki était revenu sur sa déclaration initiale, dont les militants continuaient néanmoins à lui tenir rigueur en affirmant qu'elle avait affaibli la lutte contre le SIDA et donc causé des morts inutiles.

\section{Méthode didactique}

Sans ces informations d'arrière-plan, l'interprète ne peut saisir les intentions sousjacentes qui se dissimulent «entre» les mots prononcés. Un tel dialogue peut être pris comme exemple pour inciter les étudiants à «contextualiser» le discours à traduire. On leur demandera d'abord de replacer les noms propres (Mbeki) et les abréviations (ANC, SIDA) dans leur contexte, puis de faire appel à leurs connaissances personnelles préalables pour bien cerner le sujet général abordé (le SIDA, aspects médicaux, sociaux, politiques, etc.). À l'appui des connaissances réunies sur les acteurs du dialogue et leurs parcours respectifs (par ex. rôle historique de l'ANC, positions politiques), on tentera alors de cerner ce qui constitue le contexte d'ensemble du passage à interpréter; l'étudiant verra que ces connaissances sont un récipient dans lequel le locuteur va déverser ses propres informations, mais sans lequel ces informations s'écouleraient comme de l'eau entre ses doigts.

Pour sensibiliser les étudiants à la charge cognitive des situations d'interprétation, il est proposé de leur présenter des phrases courtes ${ }^{4}$ contenant des références culturelles, historiques ou d'actualité, en leur demandant de laisser «remonter» à la 
mémoire toutes les informations (ou connotations) que ces phrases évoquent pour eux. La simple mention d'un nom propre (par ex. dans l'actualité) ou d'un événement donné (historique ou culturel) peut raviver ou structurer des connaissances indispensables à la compréhension des tenants et aboutissants d'une situation d'interprétation. Ainsi, on comprendra rapidement que l'expression américaine «9/11» n'a rien à voir avec les mathématiques, et que «to file for Chapter 11 » ne contient aucune allusion au chapitre d'une œuvre romanesque.

Dans le même ordre d'idées, il sera intéressant de lire en présence des étudiants les titres du journal du jour, en leur demandant à chaque fois s'ils sont capables de reconstituer le cadre général dans lequel chaque article se situe. Un tel exercice, prenant pour base, à titre d'illustration, un journal du 20 juillet 2004, fait ainsi apparaître les titres ${ }^{5}$ et les domaines suivants ${ }^{6}$ :

Les héros du putsch manqué contre Hitler célébrés (thèmes à développer: le nazisme, la résistance à Hitler);

Europol: l'UE donne son feu vert à l'accord avec la Suisse (thèmes à développer: les négociations commerciales et politiques entre la Suisse et l'UE, la coopération des polices au plan international);

Roche trouve en Bayer l'acheteur idéal pour ses produits en vente libre (thèmes à développer: le fonctionnement des grands laboratoires et entreprises pharmaceutiques, les fusions d'entreprises);

Les négociateurs entament une course contre la montre à l'OMC (thèmes à développer: le fonctionnement de l'OMC, les négociations commerciales internationales et leurs enjeux);

Après avoir longtemps fermé les yeux, les États-Unis font la chasse aux dopés (thèmes à développer: le dopage dans le sport, le CIO, l'AMA et les Fédérations internationales); Un séminariste autrichien inculpé pour images pédophiles (thèmes à développer: la pédophilie, la position de l'Église catholique en la matière);

Les victimes de Bhopal seront indemnisées (thèmes à développer: l'accident de Bhopal, les entreprises multinationales, la protection de l'environnement, la responsabilité civile et pénale des entreprises).

\section{Valeur pédagogique}

Cette méthode permet aux étudiants de constater par eux-mêmes l'étendue, par définition individuelle et variable, de leurs connaissances et de leurs lacunes, de prendre conscience de l'importance des sujets d'actualité pour l'exercice futur de la profession et d'ouvrir leur esprit à des domaines qu'ils pourraient avoir tendance à négliger de par leurs affinités personnelles (macro-économie, vie des entreprises, technique, médecine, sport, etc.). D'autre part, elle fait bien ressortir la prédominance du contenu sur la forme. Au terme de l'échange de vues et de la discussion des sujets étudiés, l'enseignant montrera aux étudiants l'utilité de cet exercice dans la perspective (future) d'un travail d'interprétation (passage d'une langue à une autre), où l'acte de traduire/interpréter ne peut pas se dérouler sans ces connaissances préalables.

«L'étudiant à qui on donnerait à analyser une information sans exiger au préalable qu'il mobilise tout ce qu'il sait du thème qu'on lui indique, n'en entendrait que les mots et serait poussé à les traduire tels quels» (Seleskovitch et Lederer 1989). Précisons bien que notre exercice n'a pas pour but de développer la maîtrise de connaissances en tant que telles, mais de préparer le terrain à la mise en place de cadres de référence généraux, à l'intérieur desquels chaque orateur vient ensuite exposer ses 
propres opinions, son propre éclairage, sa propre personnalité. On insistera à l'aide d'exemples concrets tirés de la réalité professionnelle sur l'importance de saisir les tenants et les aboutissants d'une situation de communication donnée, pour que l'interprète soit mieux à même d'en connaître les enjeux.

L'activation de ces connaissances préalables remplit un rôle essentiel. "La communication peut [...] être décrite comme étant un processus de reconnaissance par inférence des intentions de la personne qui communique» (Degueldre 2002). Ou encore: «La compétence linguistique ne joue jamais seule dans l'interprétation d'une phrase réelle; il y faut toujours une part de connaissances qui n'est pas rattachée directement à l'énoncé» [...] "S'il y a identité entre l'information reçue et la connaissance antérieure, le rattachement est direct et la compréhension équivaut à une re-connaissance» (Seleskovitch 1968). D'autre part, ces connaissances font boule de neige. "C'est un fait curieux que, loin de diminuer l'intérêt, la connaissance l'augmente, et qu'à tout intérêt en éveil correspond l'acquisition de connaissances nouvelles» (Séleskovitch 1968). Ainsi peut-on espérer déclencher chez les étudiants un processus qui s'auto-alimente et entraîne une véritable «boulimie » de connaissances, non pour elles-mêmes, mais pour leur valeur dans une possible situation d'interprétation future.

\section{Hiérarchisation}

La deuxième étape de l'enseignement consiste à révéler de manière éloquente et sensible la nécessité de travailler sur le sens exprimé et sur l'organisation des idées plutôt que sur la forme superficielle du discours. Sans vouloir entrer dans le débat de fond beaucoup plus large relatif à la notion de «sens», précisons qu'il s'agit surtout de montrer aux étudiants que l'interprétation est d'abord et avant tout une compréhension. Quels que soient les processus cognitifs et intellectuels en jeu, tout énoncé oral suscite une perception suivie d'un décodage qui active dans le cerveau de l'auditeur (ici, de l'interprète) les zones et combinaisons de zones lui permettant de dégager une signification des propos entendus. Or, l'expérience montre qu'un étudiant à qui on demande d'interpréter une courte intervention a parfois tendance à se concentrer sur les mots au détriment du sens général, qu'il aurait pourtant aisément pu dégager s'il avait su que cette écoute ne serait pas suivie d'une interprétation! Cette forme d'incertitude de Heisenberg (soit on comprend, soit on interprète!) demeure heureusement la prérogative des étudiants débutants qui n'ont pas encore compris tout le processus.

\section{Méthode didactique}

On donnera connaissance aux étudiants d'un ensemble d'informations factuelles comportant, sans ordre particulier, et même de préférence dans le désordre, un grand nombre de détails d'importance variable. Puis, au lieu de les inviter à interpréter, à mémoriser ou à répéter, on les invitera à hiérarchiser les idées entendues. On commencera par dégager le thème du discours (de quoi parle-t-on), puis le rhème (qu'en dit-on), puis la succession éventuelle des idées avec leur enchaînement logique, et enfin les détails concrets (dates, chiffres, lieux, noms propres, etc.). Le texte ci-dessous peut servir d'exemple à une telle approche. 
Grande-Bretagne. La découverte au bord d'une route de plans confidentiels visant à protéger d'un attentat Heathrow, le principal aéroport de Londres, a relancé lundi la crainte d'une menace terroriste jugée très crédible au Royaume-Uni. Scotland Yard avait annoncé dimanche soir l'ouverture d'une enquête, après qu'un automobiliste eut retrouvé les plans. Les documents détaillaient notamment les horaires de patrouille, les déploiements des tireurs d'élite sur les toits, les fermetures de routes et les itinéraires que les terroristes pourraient emprunter après une attaque ${ }^{7}$.

On dégagera tout d'abord le thème (la lutte contre le terrorisme), qu'il conviendra, dans le droit fil de l'exercice précédent, de replacer dans son contexte plus large (attentats du 11 septembre, guerre en Irak, coalition anglo-américaine, etc.). On spécifiera ensuite le rhème (risques concrets en Grande-Bretagne). De proche en proche, on s'attachera à structurer le discours en accordant aux idées un ordre d'importance décroissant ou chronologique (par ex. existence de plans d'actes terroristes [avec liste: horaires, tireurs d'élite, fermeture de routes, itinéraires], vol de ces plans, imminence de la menace), en laissant pour la fin les détails (bord de route, aéroport de Heathrow, lundi, Scotland Yard, dimanche soir, automobiliste).

\section{Valeur pédagogique}

Cet exercice revêt une très grande importance et sa position dans les toutes premières étapes de l'apprentissage de l'interprétation de conférence ne saurait être décalée, vu le risque considérable que les traditionnels exercices de mémorisation ne viennent renforcer les mauvaises habitudes d'écoute des étudiants, en axant leur concentration sur les mots entendus plutôt que sur la structure des idées. En effet, l'observation première que fait l'enseignant qui confronte les étudiants à une situation de «translation » est que la propension à s'accrocher aux mots est une seconde nature contre laquelle il convient de lutter toujours et sans cesse. Les premiers mots d'un discours ne sont pas toujours les plus importants, ni les derniers les plus secondaires. Il est donc essentiel de ne pas s'attacher à ce qui semble le plus frappant, pour se concentrer délibérément sur ce qui est le plus signifiant.

D'autre part, un tel exercice jette les bases de deux points essentiels de la prise de notes en consécutive: la symbolisation et la hiérarchie des idées. Si les étudiants parviennent à écouter un discours du type de celui présenté plus haut pour en dégager quelques idées-force assorties de quelques détails dûment hiérarchisés, la notation ultérieure de ces idées se fera naturellement, à la fois par des graphèmes succincts ou symboliques et selon une représentation faisant visuellement ressortir l'importance respective des diverses idées (Rozan 1956). On utilisera ensuite des textes ou discours présentant une argumentation serrée, avançant d'une idée à l'autre par concessions, digressions, déductions, oppositions, illustrations, etc., afin de faire ressortir 'tout nu' le squelette de l'intervention. Les étudiants apprennent ainsi, puis mettent en œuvre un mode de pensée qui encourage systématiquement l'analyse des propos.

Cet exercice doit être expliqué, décortiqué, puis répété le plus souvent possible afin de devenir une seconde nature. Ce n'est qu'une fois ce stade atteint et bien maîtrisé qu'il sera utile de commencer les exercices de mémorisation, car ceux-ci pourront alors inciter les étudiants à retenir le sens du discours et non sa formulation. 


\section{Images mentales}

«Parmi les premiers moyens qu'il convient de recommander aux étudiants pour les concentrer sur le sens et les empêcher de s'attarder sur les mots, figure la visualisation. Voir mentalement un objet ou imaginer les aspects d'un événement dont on entend la description, c'est en entendre le sens» (Seleskovitch et Lederer 1989).

La phrase: "Au début du siècle, les familles italiennes avaient de nombreux enfants qui émigraient dans les pays les plus divers pour trouver une situation » a été traduite ainsi par un débutant: «All'inizio del secolo, numerose famiglie emigravano in diverse paese per trovare una occupazione.» Le simple terme de "nombreux» avait été pris "au pied de la lettre», sans que l'étudiant ne se rende compte qu'il s'appliquait aux enfants et non aux familles. Nous avons tout lieu de croire que l'étudiant s'est appliqué à reproduire aussi fidèlement que possible (croyait-il) la phrase initiale (en faisant appel à un effort de mémorisation superficielle), sans s'en représenter mentalement le contenu. Au terme de son effort de mémoire, seule la «coquille» lexicale du mot «nombreux» lui est revenue, alors qu'une image concrète lui aurait permis d'éviter une erreur de sens. «De nombreux exercices de visualisation sont de mise; car l'habitude d'écouter des mots au lieu de voir les images qu'ils évoquent est bien ancrée» (Seleskovitch et Lederer 1989).

\section{Méthode didactique}

Pour chaque exemple utilisé en cours, on invitera les étudiants à se représenter une image mentale très concrète. On partira de notions simples, par exemple « une chemise bleue», puis en insistant sur l'importance de "voir» mentalement l'objet (le vêtement) ainsi que ses caractéristiques (la couleur) avant de procéder à une quelconque traduction, afin d'éviter les correspondances que l'on pourrait penser «automatiques ». Les couleurs donnent d'ailleurs une excellente occasion de montrer les pièges des équivalences lexicales superficielles. Ainsi, le coréen et le japonais décrivent comme «bleue» la couleur du feu de circulation autorisant le passage (Choi 2003). Si l'interprète qui travaille par exemple vers l'anglais ou le français ne se représente pas mentalement une situation précise (un carrefour avec feux de circulation tricolores), il produira une phrase digne de la pire traduction informatisée que l'on puisse trouver sur Internet... et se couvrira de ridicule. En effet, le rôle de l'interprète dans une telle circonstance est de se concentrer sur l'intention (Reiss 1984; Gile 2003) recherchée par la mention du feu «bleu»: en l'occurrence, cette couleur est celle qui, parmi les trois couleurs possibles, se trouve en bas (ou à droite) et symbolise l'autorisation de franchir le carrefour. C'est cette notion-là (la liberté de passage), et non la couleur en tant que telle, qu'il convient de traduire, dans le respect des réalités et des codes culturels de la communauté des locuteurs de la langue d'arrivée.

On utilisera ensuite des notions plus abstraites, par ex. "une entreprise», en invitant les étudiants à trouver, coûte que coûte, un moyen de visualiser concrètement cette notion générale (par exemple, un gratte-ciel en verre symbolisant le siège social). De proche en proche, on formera des phrases qui seront soumises au même exercice. Exemple: "Cette année, l'entreprise Coca-Cola a dégagé un bénéfice de 10 milliards de dollars. » La représentation mentale pourrait associer un gratte-ciel, le célèbre logo de Coca-Cola et des quantités importantes de billets de banque. Là encore, plus 
les phrases seront longues ou complexes, plus on pourra y associer l'exercice de hiérarchisation décrit plus haut.

On pourra même systématiser l'exercice en tentant de susciter des images mentales, même pour des notions très abstraites ou philosophiques ( "la générosité»: un individu distribuant des aumônes autour de lui ; «la maladie» : un patient dans un lit d'hôpital; "la persuasion»: un vendeur dans un magasin, etc.). Le but n'est pas réellement de vouloir à tout prix assortir chaque mot, chaque formulation, chaque phrase, d'un équivalent susceptible d'être peint ou dessiné. Il est plutôt, par ce biais assez parlant pour l'imagination, de donner libre cours à la liberté de chacun face à ce qu'il entend et comprend, car cette liberté de «tourner autour du sens» est aussi le premier pas vers la liberté de trouver, dans la langue cible, une formulation appropriée et échappant à l'influence de la langue source.

\section{Valeur pédagogique}

De proche en proche, on prendra même des exemples d'expressions idiomatiques ou de proverbes, censés par définition évoquer des situations générales à l'aide de formulations stéréotypées, dans le but d'inciter les étudiants à en comprendre le sens global et les divers contextes d'utilisation possibles. Ainsi, la "visualisation» de la phrase «It is better to light a candle than to curse the darkness » mettra en présence deux individus, l'un, maussade et maugréant parce qu'il ne trouve pas son chemin dans le noir, l'autre réussissant à s'orienter grâce à une modeste chandelle. En rétablissant un certain degré d'abstraction, on fera ressortir l'idée qu'un petit effort entrepris avec optimisme vaut mieux qu'une manifestation de défaitisme passif, que tout acte concret, même modeste, contribue au progrès, etc. Dès lors, l’idée générale de la valeur de l'optimisme et de la lutte contre le découragement pourra s'exprimer librement, par les moyens linguistiques et stylistiques de chacun, y compris dans une langue différente de la langue de départ, et par exemple, en français, par la célèbre citation: "Il n'est pas nécessaire d'espérer pour entreprendre ni de réussir pour persévérer ${ }^{8}$ »

La représentation mentale des idées et des concepts en vue de l'interprétation a fait l'objet d'un récent article dont la partie théorique constitue un rappel éclairant des principes de la compréhension (Degueldre 2002). Notre thèse personnelle consiste à introduire dans l'enseignement de l'interprétation cette activité intellectuelle comme un exercice délibéré, mais non comme une fin en soi. Il n'est pas nécessairement utile que tous les interprètes débutants se forcent à tout visualiser en termes concrets, sous prétexte que l'imagerie mentale est utile; il suffit qu'ils aient fait l'expérience ponctuelle de son utilité pour la compréhension d'un message. À eux ensuite de mettre cette technique à profit de manière systématique ou non, de l'appliquer à des textes abstraits ou non, de s'entraîner ou non à la mettre en œuvre, etc. Le parallélisme avec l'utilisation des symboles ou des dessins dans la prise de notes en consécutive s'impose: l'utilité et la valeur des symboles ne font aucun doute, mais chacun doit rester libre d'en faire un usage modéré ou immodéré dans chaque situation concrète. Il n'en reste pas moins qu'une application bien comprise peut faire des miracles, surtout (à un stade ultérieur de l'apprentissage) face à des discours difficiles, abstraits ou touffus, dont l'accès passe par une «clef»: cette méthode en est une. Prenons à titre d'exemple conclusif un extrait de texte utilisé lors d'une récente 
session d'examen à l'ÉTI', et l'on verra comment la combinaison des trois exercices décrits plus haut permet de surmonter des difficultés importantes (il est bien entendu que, sur le plan pédagogique, ces difficultés doivent être réservées aux étudiants en fin de cursus).

Diese Ausprägung einer zur Zivilreligion überhöhten Politik hat zum Zusammenhalt der aus dem beständigen Strom an Einwanderern unterschiedlichster Herkunft konstituierten amerikanischen Nation beigetragen.

La contextualisation permet de faire ressortir toutes les informations préalables requises de l'interprète: esquisse de l'histoire des États-Unis, rôle des immigrants, considérations politiques actuelles. La hiérarchisation des idées permet de donner à la notion de "Zusammenhalt» toute la valeur qui lui revient, suivie de celles de «Zivilreligion» et de «Politik», puis des informations qualifiant la nation américaine ( Einwanderer»), elles-mêmes précisées à titre secondaire et complémentaire par les notions de «beständiger Strom» et de «unterschiedlichster Herkunft». Enfin, la visualisation permet de se représenter une convention démocrate ou républicaine (avec majorettes, musique, orateurs et une foule "chauffée à blanc»), que l'on n'aura pas de mal à comparer à une cérémonie religieuse (visite du pape en Pologne, par exemple). La mise en commun des efforts cognitifs conjoints autour de ces trois axes en vue d'analyser cette phrase facilitera grandement le travail de l'interprète, pour lequel par exemple la «traduction» du terme abstrait de "Ausprägung» deviendra un problème secondaire (et même ne se posera plus du tout). Il lui sera dès lors aisé de trouver une formulation efficace et pertinente $^{10}$.

\section{Conclusion}

Les trois types d'exercices pédagogiques que nous avons développés ici sont destinés à «apprivoiser» la tâche a priori écrasante de l'interprète en disséquant autant que faire se peut les divers processus mentaux impliqués ${ }^{11}$, en assurant une progression lente, mais rigoureuse, en associant systématiquement chaque étape du processus à l'acquisition de compétences indispensables pour l'étape suivante, et en n'aboutissant qu'en dernier lieu à la mise en regard de deux langues différentes. Le travail sur le contexte, puis sur la structure et enfin sur la visualisation présente l'avantage de pouvoir être effectué en rapport avec une culture donnée plutôt qu'avec une langue déterminée. Enfin, la réalisation d'exercices impliquant le passage d'une langue à une autre intervient à un moment où l'interprète débutant a assimilé de «bons » réflexes lui permettant de ne pas s'obnubiler sur le transfert lexical et de se concentrer sur l'objectif de communication et de transfert culturel poursuivi par l'acte d'interpréter.

\section{NOTES}

1. Nous n'entrerons pas ici dans la querelle concernant l'ordre de l'enseignement de la simultanée et de la consécutive, mais nous appuierons seulement sur la pratique de l'Université de Genève.

2. "[...] Interpreting students readily accept the idea that the right way to interpret is to try to understand what the speaker is trying to say" (p. 51).

3. "Briefly, these steps involve (1) listening and memory exercises, (2) shadowing, (3) dual-task training, (4) paraphrasing, (5) abstracting, (6) clozing, (7) sight translation, (8) sight interpretation, (9) processing of digits, proper names, technical words and acronyms, (10) lagging exercises, (11) anticipation exercises and (12) left- and right-ear processing exercises" (Ilg \& Lambert, 1996, p. 76). 
4. D. Séleskovitch soulignait l'importance de s'abstenir de phrases trop courtes, pour utiliser des passages relativement longs afin d'éviter le calque linguistique. Soulignons bien qu'elle se plaçait dans le cadre d'un exercice de restitution (sans prise de notes), alors que nous insistons ici sur un travail cognitif préalable à toute tentative de passer d'une langue à une autre.

5. Journal Le Temps du 20 juillet 2004.

6. Nous laisserons de côté les informations purement factuelles ou liées à une actualité immédiate, pour ne retenir que celles qui évoquent une réalité culturelle ou historique susceptible d'être mentionnée dans d'autres circonstances faisant appel à des interprètes de conférence.

7. Journal Le Temps 20 juillet 2004.

8. Attribuée à Guillaume le Taciturne.

9. Discours de M. Karsten D. Voigt, coordonnateur chargé de la coopération germano-américaine, université Georg August, Göttingen, le 11 mai 2004, sous le titre «Deutsch-amerikanische Beziehungen - Krise oder Neuanfang?» Examen de diplôme, épreuve d'interprétation simultanée avec texte, session de juin/juillet 2004.

10. Par exemple: Cette politique portée au rang de religion civile contribue à la cohésion de la nation américaine, qui est le produit d'un afflux incessant d'immigrants d'origines les plus variées.

11. Les efforts engagés par K. Dejean Le Féal pour fragmenter les difficultés en interprétation simultanée partent eux aussi précisément du constat selon lequel tout apprentissage de cette technique court un grand risque d'échec (Dejean Le Féal 1997) s'il ne s'accompagne pas d'une tentative de délimiter clairement les diverses étapes de la progression, afin de les surmonter l'une après l'autre.

\section{RÉFÉRENCES}

Cног, J. (2003) : «Interpreting Competence and Cultural Differences», Forum 1-1, Paris, Sorbonne nouvelle.

Clifford, A. (2001): «Discourse Theory and Performance-Based Assessment: Two Tools for Professional Interpreting», Meta 46-2, p. 365-378.

Degueldre, C. (2002), «Le rôle de l'imagerie dans la communication réalisée par un interprète », Meta 47-1, p. 58-86.

Dejean Le Feal, K. (1997), «Simultaneous Interpretation with “Training Wheels” ", Meta 42-4, p. 616-621.

Gile, D. (2003) : «Justifying the Deverbalization Approach in the Interpreting and Translation Classroom », Forum 1-2, Paris, Sorbonne nouvelle.

ILG, G. and S. LAmbert (1996) : «Teaching Consecutive Interpreting”, Interpreting 1-1, Amsterdam/ Philadelphia, John Benjamins, pp. 69-99.

Kalina, S., Buhl, S. und H. Gerzymisch-Arbogast (éds) (2000): Dolmetschen: Theorie, Praxis, Didaktik, St. Ingbert, Röhrig Universitätsverlag.

Lambert, S. and B. Moser-Mercer (éds) (1994) : Bridging the Gap. Empirical Research in Simultaneous Interpretation, Amsterdam/Philadelphia, John Benjamins.

Pöchнаскеr, F. (1994): Simultandolmetschen als komplexes Handeln, Tübingen, Günter Narr Verlag.

Reiss, K. und H. Vermeer (1991): Grundlegung einer allgemeinen Translationstheorie, Tübingen, Niemeyer.

Roy, C. B. (2000) : Interpreting as a Discourse Process, Oxford/New York, Oxford University Press. Rozan, J. F. (1956): La prise de notes en interpretation de conference, Genève, Georg.

Seleskovitch, D. (1968): L'interprète dans les conférences internationales, Paris, Lettres modernes. Seleskovitch, D. and M. Lederer (1989): Pédagogie raisonnée de l'interprétation, Paris, Didier. Seleskovitch, D. and M. Lederer (1993): Interpréter pour traduire, Paris, Didier. 